\title{
A Resilient, Untethered Soft Robot
}

\section{Citation}

Tolley, Michael T., Robert F. Shepherd, Bobak Mosadegh, Kevin C. Galloway, Michael Wehner, Michael Karpelson, Robert J. Wood, and George M. Whitesides. 2014. "A Resilient, Untethered Soft Robot." Soft Robotics 1 (3) (September): 213-223. doi:10.1089/soro.2014.0008.

\section{Published Version}

doi:10.1089/soro.2014.0008

\section{Permanent link}

http://nrs.harvard.edu/urn-3:HUL.InstRepos:16920697

\section{Terms of Use}

This article was downloaded from Harvard University's DASH repository, and is made available under the terms and conditions applicable to Open Access Policy Articles, as set forth at http:// nrs.harvard.edu/urn-3:HUL.InstRepos:dash.current.terms-of-use\#OAP

\section{Share Your Story}

The Harvard community has made this article openly available.

Please share how this access benefits you. Submit a story.

Accessibility 


\section{A Resilient, Untethered Soft Robot}

Michael T. Tolley ${ }^{1,2}$, Robert F. Shepherd ${ }^{3,4}$, Bobak Mosadegh ${ }^{2,3}$, Kevin C. Galloway ${ }^{1,2}$, Michael Wehner $^{1,2}$, Michael Karpelson ${ }^{1,2}$, Robert J. Wood ${ }^{1,2, *}$, and George M. Whitesides ${ }^{2,3, *}$

${ }^{1}$ School of Engineering and Applied Sciences, Harvard University

60 Oxford Street, Cambridge, MA 02138

${ }^{2}$ Wyss Institute for Biologically Inspired Engineering, Harvard University

3 Blackfan Circle, Boston, MA 02115

${ }^{3}$ Department of Chemistry and Chemical Biology, Harvard University

12 Oxford Street, Cambridge, MA 02138

${ }^{4}$ School of Mechanical and Aerospace Engineering, Cornell University

105 Upson Hall, Ithaca, NY 14853

*Corresponding authors,

email: gwhitesides@gmwgroup.harvard.edu

email: rjwood@eecs.harvard.edu

Target Journal(s): Advanced Materials, Advance Functional Materials

Keywords: soft robots, soft composite materials, untethered, pneumatic, bioinspired 


\begin{abstract}
A pneumatically powered, fully untethered mobile soft robot is described. Composites consisting of silicone elastomer, polyaramid fabric, and hollow glass microspheres were used to fabricate a sufficiently large soft robot to carry the miniature air compressors, battery, valves, and controller needed for autonomous operation. Fabrication techniques were developed to mold a 0.65 meter long soft body with modified Pneu-Net actuators capable of operating at the elevated pressures (up to $138 \mathrm{kPa}$ ) required to actuate the legs of the robot and hold payloads of up to $8 \mathrm{~kg}$. The soft robot is safe to handle, and its silicone body is innately resilient to a variety of adverse environmental conditions including snow, puddles of water, direct (albeit limited) exposure to flames, and the crushing force of being run over by an automobile.
\end{abstract}

\title{
1 Introduction
}

There is a nascent class of robots—-so called "soft" robots - that contain no (or few) rigid internal structural elements, and are loosely modeled on animals with non-rigid body parts (starfish, squid, and others). ${ }^{[1,2,3,4,5,6]}$ Many of these soft robots are actuated pneumatically using gas transferred to them from a stationary source via a flexible tether. ${ }^{[7,8,9,10]}$ Those we and others have described previously have not been sufficiently large (presently $\sim 15 \mathrm{~cm}$ in largest dimension), nor actuated at sufficiently high pressures ( 0.5 atm; $7 \mathrm{psi} ; \sim 48 \mathrm{kPa})$ to support the size or weight of commercially available power supplies (e.g., batteries and compressed gas cylinders) and the other components (e.g., valves, air compressors, circuit boards) necessary to operate autonomously. The limitations imposed by size are one primary reason that they have been designed to function with compressed gas supplied through an external pneumatic tether. Although this tether may interfere with some tasks, it is often an advantage rather than a 
disadvantage for others: for example, it enables the transfer or sampling of fluids and solids ${ }^{\text {[10] }}$ and facilitates electronic communication and optical observation. Nonetheless, robots intended for use outside of laboratory environments should be able to operate without the constraints of a tether; this is especially true for robots intended to perform demanding tasks in challenging environments (for example, for search and rescue applications in unstable rubble).

We have developed composite soft materials, a mechanical design, and a fabrication method that enable the untethered operation of a soft robot. This robot can operate in two modes: using a battery pack (for several hours), and using a very light-weight electrical tether (for much longer periods). Though these robots are composed primarily of synthetic elastomers, we provide several demonstrations that they are capable of operating outside a forgiving laboratory environment: in a snowstorm, in puddles of water, and in direct (albeit limited) exposure to flames.

We fabricated a quadrupedal soft robot $\sim 0.65$ meters in length (Figure 1a; Figure S2) that can be driven for two hours on a flat surface using a battery pack $(3,200 \mathrm{mAh}$, lithium/polymer; E-flite) at speeds of $>18.0 \mathrm{~m} \mathrm{hr}^{-1}$ in a walking gait, and $>2.0 \mathrm{~m} \mathrm{hr}^{-1}$ in an undulating gait. The design of the robot was based on that of a previous, tethered quadrupedal soft robot. ${ }^{[7]} \mathrm{We}$ modified four characteristics of the tethered robot in order to develop an untethered one that is resilient to a variety of environmental conditions. We (i) designed a higher strength (and lower density) composite elastomeric material for the body, so that the robot could operate at higher pneumatic pressures; (ii) designed a larger body size to accommodate and support the power source; iii) employed a modified Pneu-Net $(\mathrm{PN})$ architecture, ${ }^{[11]}$ to allow more rapid and stable actuation than our previous Pneu-Net design; and (iv) incorporated an electrically powered onboard air compressor, a system of valves, and a controller for pneumatic actuation. 
Figure 1. Designing composites for improved material properties. A) Two material composites are used in the soft robot. The top layer of the robot (black) is a blend of hollow glass spheres in silicone, and the bottom layer (white) is a Nylon mesh impregnated with the same silicone. B) The stress strain curves for the material comprising the top (glass bubbled M4601) and bottom layers (Nylon mesh w/M4601), as well as pure M4601 and Ecoflex 0030, the material used in prior soft robots for comparison.
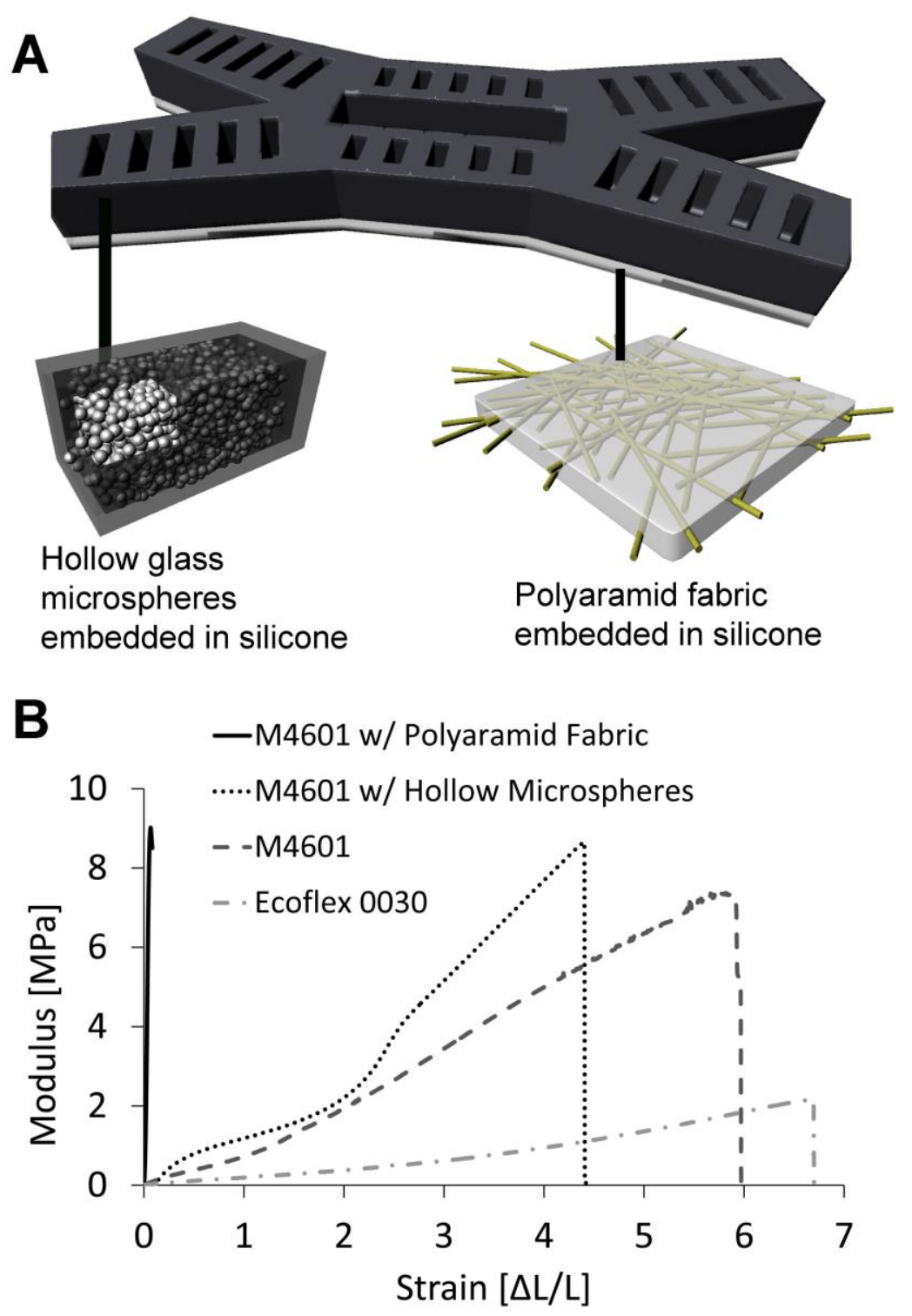
The robot is primarily composed of silicone rubber. This material has a glass transition temperature (depending on additives) of $\sim-120^{\circ} \mathrm{C}$, and thermal stability to temperatures up to $~$ $400{ }^{\circ} \mathrm{C} .{ }^{[12]}$ Silicone soft robots are thus, in principle, capable of operating in environments in which temperature has a wide range. In addition, many siloxane derived polymers are: (i) hydrophobic (i.e., water resistant) and energetically stable to corrosive, nucleo- and electrophilic attack from many polar moeties; ${ }^{[13]}$ (ii) resistant to ultraviolet (UV) light, and thus stable in intense sunlight over several decades; ${ }^{[14]}$ and (iii) fire resistant, so capable of surviving brief, but direct, exposure to flames. ${ }^{[15,16]}$

\section{Experimental Design}

The design presented here is a prototype of an untethered system that incorporates a complete set of functional elements (body, power source, control system, and sensors). The selection of these elements resulted from (and in) a set of empirical tradeoffs, and certainly does not yet represent a fully optimized set for any specific application. It was designed, instead, to give a starting point for the development of a family of untethered soft robots.

\subsection{Material selection/design of material composites}

To carry the increased load of the pneumatic pumps and control electronics, as well as a body larger than that of our prior quadrupedal robots, ${ }^{[7]}$ we used a higher elastic modulus silicone ( 7.0 MPa or 1,015 psi; M4601, Wacker Chemicals; Figure 1) with similar extensibility ( 700\% strain to failure) in order to actuate with higher pressures as the silicone elastomer used previously $^{[7,8]}$ ( 0.11 MPa or 16 psi; Ecoflex 0030, Smooth-On; Figure 1).

We reduced the weight of the robot's body by $\sim 40 \%$ relative to scaled versions of previous designs by incorporating hollow glass spheres into the silicone (Supplemental Information, SI, contains experimental details). The addition of the glass spheres reduced the extensibility of the 
material (to $\sim 400 \%$ strain to failure), but this reduction did not compromise the operation of the robot due to the improved mechanical design of the actuators. ${ }^{[11]}$

\subsection{Power source}

We explored the advantages and disadvantages of using compressed gas sources and electrically operated air compressors to provide pneumatic actuation of the robot. In summary, compressed gases provide higher flow rates, but for shorter durations than mini air compressors. For applications such as search and rescue, we determined that electrically powered mini air compressors are the best choice (the SI contains details concerning comparisons of power sources). A major benefit of on-board air compressors is that they can be operated interchangeably either tethered (with thin, light-weight, and flexible electric wires) or autonomously (for up to two hours via battery with the tether detached).

\subsection{Body architecture and fabrication}

The body of our soft robot consists of four legs connected to a central body, each of which is actuated by a Pneu-Net, in a configuration identical to our previous tethered quadrupedal soft robot design (Figure 2a). ${ }^{[7]}$ In order to increase the rate of actuation of the larger untethered robot, we used a Pneu-Net design that allows for actuation at lower pressures, and with less volumetric flow of gas into the Pneu-Nets, than our prior design (Figure 2b). ${ }^{[1]}$ The spine of the robot is actuated by two parallel Pnet-Nets with space between them to accommodate the power supply, control board, and two air compressors (Figure 2c; systems diagram of compressors and valving shown in Figure S1).

Due to the large size of the robot $(\sim 0.65 \mathrm{~m}$ in length), we found the use of molds assembled from pieces of laser-cut sheets of $6 \mathrm{~mm}$ thick acrylic to be an economical and flexible option. Using integrated alignment features, we assembled the cut acrylic pieces into a three dimensional 
Figure 2. Pneumatic actuation of untethered quadrupedal soft robot. A) Schematic representation of the components of the mini air compressor (MAC) driven, battery powered soft robot. Layer 1 consisting of six Pneu-Nets is sealed onto layer 2. B) Schematic representation of a cross-section of Pneu-Net 1 as its internal pneumatic network is pressurized. C) Photographs of Pneu-Net 1 at rest (left) and pressurized to 16 psi (middle). Photograph of electronic components (mini air compressors, battery pack, relays, and circuit boards) between Pneu-Nets 3 and 4 (right) that drive the robot.

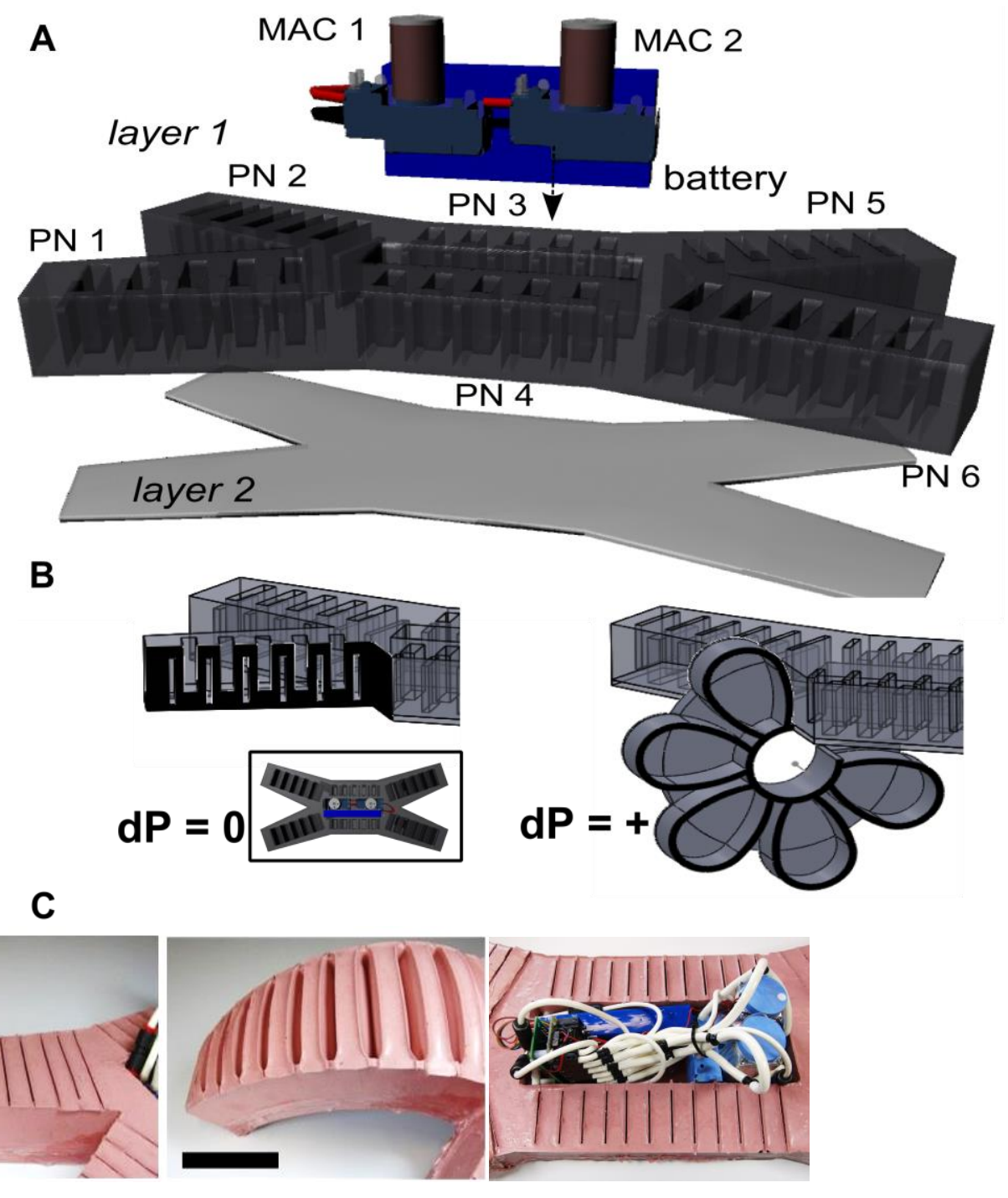


mold (see Figure S2). The modular nature of these molds greatly facilitated demolding of the cast robots. We used a water jet system to cut aluminum for the thinnest pieces of the mold, as they were most susceptible to fracture during demolding (SI Text; Figure S2).

\subsection{Control system and sensors}

The control system of the robot consisted of a custom-built control board with outputs for two mini air compressors (MACs), and six two-way valves (X-Valve, Parker Hannifin Corporation), one for each of the six PNs that actuated the robot. The MACs provided a source of pressurized air at a constant rate, while the valves switched the connection of each Pneu-Net between this elevated pressure and atmospheric pressure. One MAC supplied Pneu-Nets 2 and 3, while the other MAC supplied the remaining Pneu-Nets (i.e., Pneu-Nets 1,4,5, and 6). To actuate one of the Pneu-Nets, the corresponding valve was opened to connect the Pneu-Net to the output of the associated MAC (as well as to any other Pneu-Net currently being actuated). While a Pneu-Net was not being actuated, it was by default being vented to the atmosphere. (A "hold" state was unnecessary for the patterns of pressurization and depressurization we used for actuation of the quadruped). Using a custom-designed control board allowed us to minimize the size and mass of the control system (see SI for more details on the control system).

Control programs were stored in the onboard memory of the controller. These programs, written and uploaded using the Arduino interface, consisted of sequences of commands to the control valves and air compressors (the MACs ran at a constant voltage). The extent of actuation of a Pneu-Net was controlled by the duration the valve connecting it to the source of pressurized gas was opened.

We used a light-weight camera with audio and video recording and transmission capabilities (GoPro Hero2, Woodman Labs) as sensor when audio and visual observations were required. 


\section{Results}

\subsection{Scaling considerations}

Soft lithography is a scalable molding process. We have used this method previously to fabricate a quadrupedal robot of length $\sim 15 \mathrm{~cm} ;{ }^{[7]}$ the robot described here has a length of $\sim 65$ $\mathrm{cm}$ (Figure S2). With all relative dimensions kept constant, as the length, L, of the robot increases, the weight of the robot increases as $\mathrm{L}^{3}$, and the force the actuators apply at the same $\Delta \mathrm{P}$ increases as $\mathrm{L}^{2}$ (pressure over the internal surface area of the Pneu-Nets; SI). Consequently, for a particular actuating pressure, the robot will eventually become too heavy to support its own weight, much less any additional load necessary for untethered operation. Thus, for larger robots, we must reduce their density, and/or increase their actuation pressure (to achieve greater actuating forces). Given the weight and size of the selected pneumatic actuation source, we estimated a required overall robot body length of $\sim 0.65$ meters, which made our prior material choice (Ecoflex 0030) and actuation pressure (7 psi) insufficient to carry either the body of the robot or the components for untethered operation (data not shown).

\subsection{Low density, high modulus silicone composite}

The material system we designed for the actuating layer (Figure 1; Layer 1) was low density $(0.6 \mathrm{~g} / \mathrm{cc})$, high modulus (7 MPa), resilient ( $<10 \%$ loss in stored energy during cycling; Figure

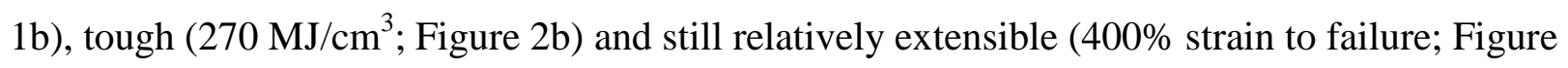
$2 b)$.

In order to prevent tears and bursting through the strain-limiting layer (Figure 1, Layer 2), we impregnated a polyaramid fabric with M4601 silicone and glued this composite sheet to the actuating layer using a thin $(<100 \mu \mathrm{m})$ layer of adhesive silicone sealant (Elastosil E 951; Wacker Chemical Corp.) spread between layers 1 and 2. In addition, to promote adhesion 
between layers, we added pegs to the bottom of layer 1; these pegs increased the surface area for bonding (Figure S2). The relative material properties of this sheet are shown in Figure 1b.

Our choices of materials and methods of assembly allowed us to make a robot that is resilient to many harsh conditions. As an example of the durability of the robot, we programmed the robot to walk underneath a Subaru Outback wagon (details on the actuation sequence used for walking are provided below), and stop with its front legs in the path of the tires of the car. After venting all of the PNs, we drove the wagon over the soft legs of the robot. Following a preprogrammed delay, the robot stood up and continued walking with no damage from the $\sim 2,000 \mathrm{~kg}(4,500 \mathrm{lbs})$ vehicle (Figure 3e-h; Video S1).

\subsection{Internal pressure capacity, load carrying ability}

The PNs that actuate the legs $(1,2,5$, and 6$)$ were able to sustain internal pressures of $\sim 172$ $\mathrm{kPa}$ (25 psi) prior to rupturing. The two PNs that actuate the spine (3 and 4) ruptured at lower pressures $\sim(152 \mathrm{kPa}$ or $22 \mathrm{psi})$, probably due to a smaller area of adhesive contact for Layer 1 and Layer 2.

Starting from a flat position, a tethered version of the soft robot was able to lift a mass of 3.4 $\mathrm{kg}(7.5 \mathrm{lbs})$ when the legs and spine were pressurized to just below their maximum tolerances (139 $\mathrm{kPa}, 20 \mathrm{psi})$. Subtracting the mass of the power and control components $(1.2 \mathrm{~kg}, 2.6 \mathrm{lbs})$, this represents a net payload capacity of $2.2 \mathrm{~kg}(4.9 \mathrm{lbs})$, or $44 \%$ of the total mass of the untethered robot (Figure S3).

Once the robot is in the standing position, the lower moment arm of a central mass on the legs meant that they could carry a larger load. With an internal pneumatic pressure of $139 \mathrm{kPa}$ (20 psi), the robot was able to hold a mass of $8.0 \mathrm{~kg}(17.6 \mathrm{lbs})$, or $160 \%$ of the total mass of the untethered robot. 
Figure 3. Untethered operation of quadrupedal soft robot. A-D) Untethered soft robot conducting indoor surveillance with onboard camera view overlaid. The robot starts moving forward with a straight ambulatory gait (A). The robot switches to a turning gait to explore a passageway on the left $(\mathrm{B}, \mathrm{C})$. The robot uses its onboard camera to image a hidden laptop (D). Green dots on the figures in the upper-right corner of each frame indicate which PNs are currently actuated (pressurized), red dots indicate unactuated PNs. E-H) Untethered soft robot operating outdoors before and after being run over by a car (E). The robot depressurizes it's actuators in preparation for impact $(\mathrm{F})$. The car running over the elastomeric legs of the soft robot $(\mathrm{G})$. The soft robot actuating and standing up after being ran over by the car $(\mathrm{H})$.
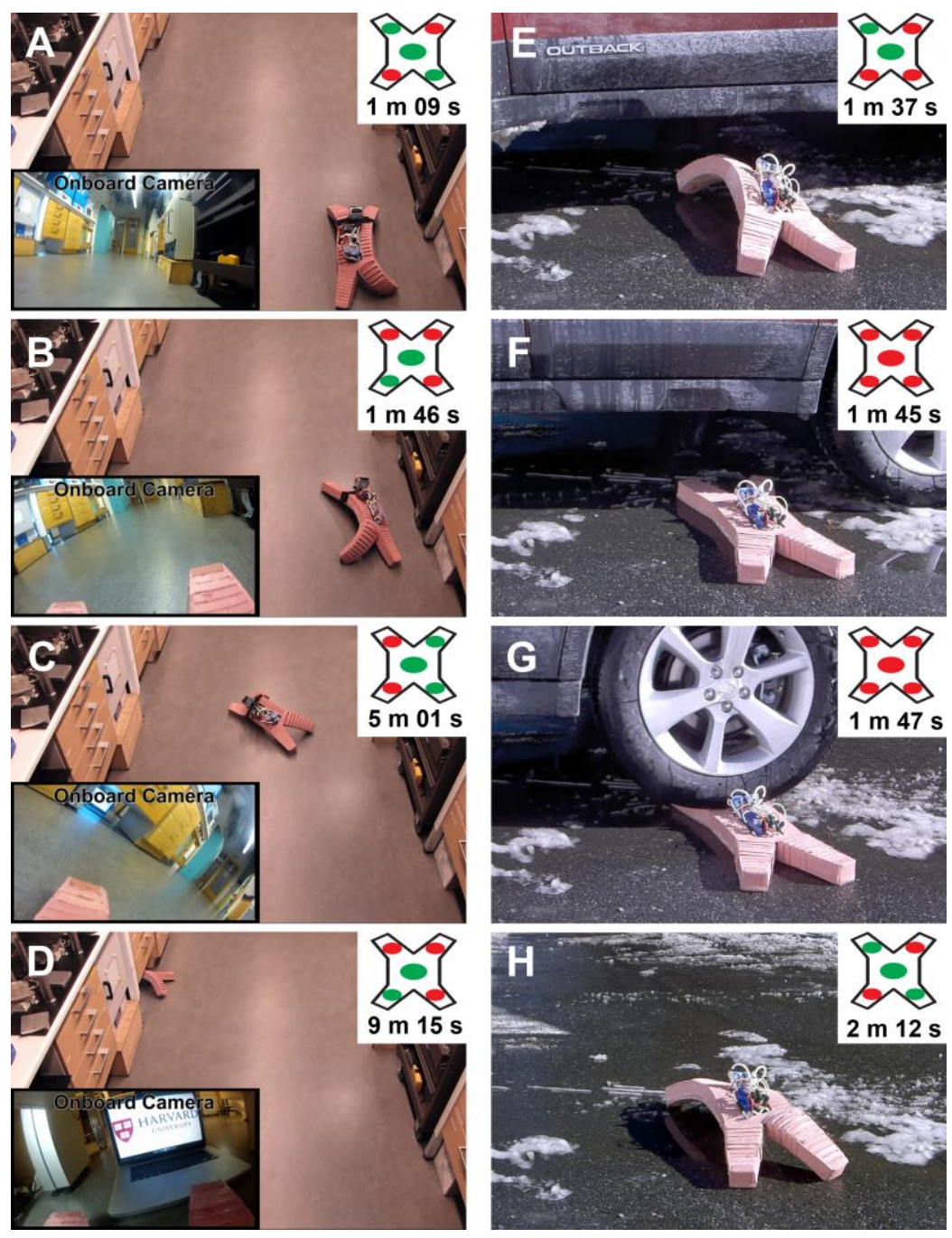


\subsection{Controls for undulating and walking gaits}

We implemented an undulating gait that actuated the Pneu-Nets of the robot in sequence, and created an actuation wave that traveled through the body from the rear toward the front; this wave resulted in forward motion (the control sequence used to accomplish this motion is described in the SI) at a velocity of $\sim 2.0 \mathrm{~m} \mathrm{hr}^{-1}$ (Figure S4, a-f; Video S2).

To command the robot in a walking gait, we used eight actuation states that caused the robot to step forward first on one side, and then the other (Figure S4, g-j; Video S2). The center PNs (3 and 4) were inflated throughout the gait to arch the back of the robot (full control sequence in SI). The walking gait allowed the robot to travel at a velocity of $\sim 18.0 \mathrm{~m} \mathrm{hr}^{-1}$.

\subsection{Remote Audio and Video Sensing: Ambulating into a small passageway (Figure 3a-d; Video S3)}

We caused the robot to turn by halving the duration of actuation of PNs 2 and 5 during the walking gait (SI). For example, in Figure 3, PNs 1 and 6 were actuated for half the duration of PNs 2 and 5. The result was a left turn with a radius of approximately $150 \mathrm{~cm}$ (a little more than two body lengths).

By strapping a forward-facing camera onto the body of the robot, we were able to perform remote audio and video sensing (here, of a laboratory, Figure 3; Video S3). For this demonstration, the information from the camera was recorded and stored on the onboard controller; the recorded audio and video were retrieved at the end of the trial.

\subsection{Resilience to harsh environments}

The material, and monolithic design, of our untethered soft robot enable it to withstand a variety of harsh environmental conditions against which traditional robots must be carefully protected (Figure 4, Video S1). 
Figure 4. Resilience of the untethered soft robot to harsh conditions. (A-C) Images from experiments of the soft robot operating untethered in a variety of harsh conditions, including a snow storm (A), a fire (B), and water (C).
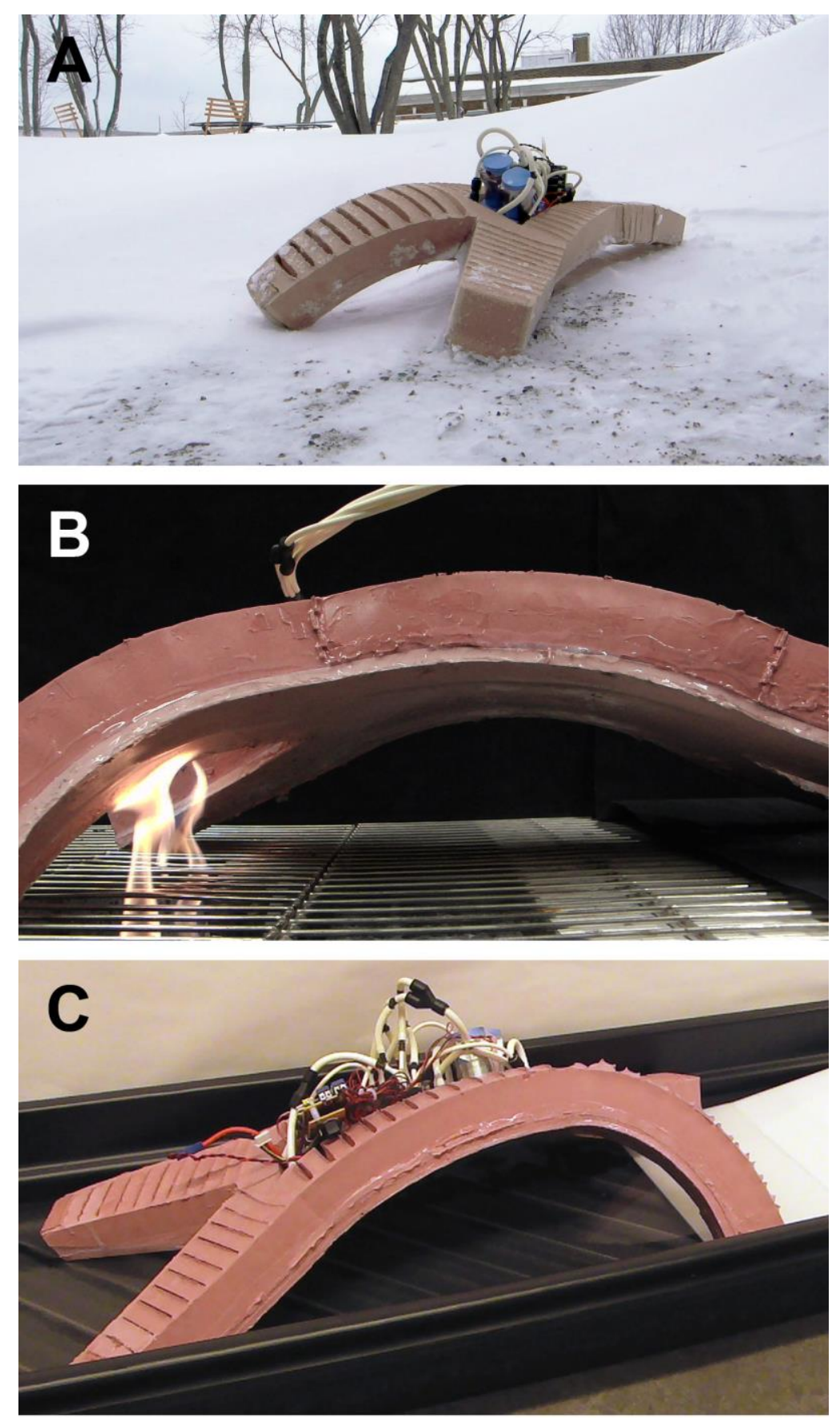
Our robot successfully executed its walking gait outside during a snowstorm (Winter Storm Nemo) with an average temperature of $-9{ }^{\circ} \mathrm{C}\left(15^{\circ} \mathrm{F}\right)$, and average wind speed of $40 \mathrm{~km} / \mathrm{h}(25$ mph, Figure 4a). Because of the low glass transition temperature of the robot's body material ( $\left.120{ }^{\circ} \mathrm{C}\right),{ }^{[12]}$ as well as the lack of sliding parts (e.g. bearings) to be contaminated, the robot ambulated normally in the snow and cold weather. Because the elastic modulus of silicone rubber is relatively constant in the range of $-20^{\circ} \mathrm{C}$ to $300^{\circ} \mathrm{C}\left(-4{ }^{\circ} \mathrm{F}\right.$ to $\left.572{ }^{\circ} \mathrm{F}\right),{ }^{[17]}$ pneumatic actuation was not impeded by the cold temperatures (although we did not develop specialized feet for travelling through snow). The robot also walked successfully in wet, slushy conditions at temperatures near $0{ }^{\circ} \mathrm{C}$ or $32{ }^{\circ} \mathrm{F}$ (Figure 3e-h; Video S1).

We commanded the robot to walk down a ramp into a plastic tray filled with $5 \mathrm{~cm}(2 \mathrm{in})$ of water (Figure $4 b$ ). The body of the robot is hydrophobic and inherently sealed against water (and is also resistant to acids ${ }^{[10,13]}$ ). It suffered no damage walking through water.

We manually controlled the ambulation of a tethered version of the soft robot across a metal grating through two flames (Figure 4c). The longest duration of direct flame exposure an element of the robot sustained was 20 seconds. Despite the exposure to extremely high temperatures $(\sim 3,000 \mathrm{~K}),{ }^{[15]}$ the robot suffered only superficial damage due to the resistance of the silicone rubber to fire and high temperatures.

\section{Discussion}

We designed a $0.65 \mathrm{~m}$ long soft robot that can carry its own weight, all the components necessary for up to two hours of untethered operation, plus an additional payload (e.g. surveillance equipment), across smooth terrain.

This resilient soft robot was enabled by the careful selection of materials and design of soft material composites. We used a silicone (M4601) that was tougher than that used previously 
(Ecoflex 0030), to support larger loads (the body of the robot, plus components for untethered operation). We added glass hollow spheres to the silicone to reduce its weight. Although the glass hollow spheres reduced the extensibility of the material, they did not reduce the toughness of the material (the integral of the stress-strain curve). Simultaneously, the new design of the Pneu-Net architecture ${ }^{[11]}$ allows the use of the slightly less extensible composite with no loss in functionality.

We demonstrated the capabilities and surprising resilience of this soft robot experimentally. We developed a turning gait and showed multi-gait capabilities in this larger soft robot, we performed untethered video reconnaissance, suitable for search and rescue missions, and we tested the robot in a range of harsh environmental conditions.

\section{Conclusion}

Silicone robots inherit the strengths of silicone rubbers, including: being impervious to water, good acid/base stability, and resistance to damage from blunt impacts or applied pressures. They are also safer in direct contact with humans. In addition, monolithic molded mechanisms (such as the body of the robot presented here) are relatively less expensive (see Table S1 for a full cost breakdown of our soft robot prototype) and less prone to failure than their assembled counterparts due to the absence of sliding parts (e.g. bearings), and reduced assembly interfaces and associated fasteners/adhesives. Pneumatically actuated soft robots do, however, also have disadvantages with respect to hard robots, including: sensitivity to large holes or tears by sharp objects, decreased precision and ability to track prescribed motions, and relatively slower actuation (although rapid actuation—100ms for actuation over a complete range of motion-is

possible with practical designs ${ }^{[11]}$ ). The balance of these advantages and disadvantages determine the suitability of pneumatically actuated soft robots for any particular application. 
The design of the soft robot presented here has the additional advantages over previous hard and soft robots of autonomy of power, good operation time between battery charges, the capabilities of audio and video sensing, and the capacity to carry larger payloads for a desired task.

One weakness of the design presented here is the sensitivity of the exposed, rigid components at the center of the robot (compressors, valves, controller, batteries) to the conditions that typically challenge rigid robots (blunt impacts, applied pressures, and harsh environmental conditions). It may be possible to alleviate this weakness by distributing the rigid components over the body of the robot and encasing them in the soft body material. Another, more technically challenging option is to replace the rigid components with soft counterparts. This approach, however, requires significant breakthroughs in the development of soft electronics, batteries, and pumps.

Another challenge with the design presented here is the slow locomotion speed of the robot, which is limited by the flow rate of air into the pneumatic actuators. This flow rate is, in turn, limited by the output pressure of the onboard compressors, as well as the flow restrictions caused by the onboard valves and tubing. Larger compressors, valves, and tubing all lead to a larger mass that must be carried by the body. Nonetheless, it may be possible to optimize these components to improve the overall speed of the robot. Alternative modes of pressurization (i.e. combustion) can be used to increase actuation speeds. ${ }^{[15]}$

Furthermore, the non-optimized feet and single degree-of-freedom legs of the robot limit it to operation on flat surfaces and at relatively low speeds. Optimization of the design of the legs and feet of the robot for locomotion would likely increase its speed and overall mobility. 
A final limitation of the current design is the requirement of preprogrammed control sequences. However, the addition of off-the-shelf bidirectional communication hardware (e.g. Xbee), would allow remote operation with live video feedback. The further development of feedback control strategies based on the available audio and/or video sensors, or additional touch, smell, or other sensors that can be integrated into the soft robot design, ${ }^{[18,19,20]}$ would allow for completely autonomous operation.

\section{Acknowledgements}

The development of the design, materials, and controls system was supported by DARPA under award number W911NF-11-1-0094. The analysis and testing of power sources was supported by Department of Energy under award number DE-FG02-00ER45852.

\section{References}

[1] D. Trivedi, C. D. Rahn, W. M. Kier, and I. D. Walker, "Soft robotics: Biological inspiration, state of the art, and future research," Appl. Bionics. Biomech., vol. 5, no. 3, pp. 99-117, 2008.

[2] K.-J. Cho, J.-S. Koh, S. Kim, W.-S. Chu, Y. Hong, and S.-H. Ahn, "Review of manufacturing processes for soft biomimetic robots," Int. J. Precis. Eng. Manuf., vol. 10, no. 3, pp. 171$181,2009$.

[3] R. Pfeifer, M. Lungarella, and F. Iida, "The challenges ahead for bio- inspired 'soft' robotics," Commun. ACM, vol. 55, no. 11, pp. 76-87, 2012.

[4] S. Kim, C. Laschi, B. Trimmer, "Soft robotics: a bioinspired evolution in robotics," Trends Biotechnol., vol. 31, no. 5, pp. 287-294, 2013.

[5] H. Lipson, "Challenges and Opportunities for Design, Simulation, and Fabrication of Soft Robots," Soft Robotics, vol. 1, no. 1, pp. 21-27, 2013. 
[6] C. Majidi, "Soft robotics: A perspective—current trends and prospects for the future," Soft Robotics, vol. 1, no. 1, pp. 5-11, 2013.

[7] R. F. Shepherd, F. Ilievski, W. Choi, S. A. Morin, A. A. Stokes, A. D. Mazzeo, X. Chen, M. Wang, and G. M. Whitesides, "Multigait soft robot," Proc. Natl. Acad. Sci. U. S. A., vol. 108, no. 51, pp. 20400-20403, 2011.

[8] F. Ilievski, A. D. Mazzeo, R. F. Shepherd, X. Chen, and G. M. Whitesides, "Soft robotics for chemists," Angew. Chem., vol. 123, no. 8, pp. 1930-1935, 2011.

[9] A. A. Stokes, R. F. Shepherd, S. A. Morin, F. Ilievski, and G. M. Whitesides, “A hybrid combining hard and soft robots," Soft Robotics, vol. 1, no. 1, pp. 70-74, 2013.

[10] R. V. Martinez, J. L. Branch, C. R. Fish, L. Jin, R. F. Shepherd, R. Nunes, Z. Suo, and G. M. Whitesides, "Robotic tentacles with three-dimensional mobility based on flexible elastomers," Adv. Mater., vol. 25, no. 2, pp. 205-212, 2013.

[11] B. Mosadegh, P. Polygerinos, C. Keplinger, S. Wennstedt, R. F. Shepherd, U. Gupta, J. Shim, K. Bertoldi, C. J. Walsh, and G. M. Whitesides, "Pneu- matic networks for soft robotics that actuate rapidly," Adv. Funct. Mater. (in press).

[12] P. Zheng and T. J. McCarthy, "Rediscovering silicones: Molecularly smooth, low surface energy, unfilled, uv/vis-transparent, extremely cross- linked, thermally stable, hard, elastic PDMS," Langmuir, vol. 26, no. 24, pp. 18585-18590, 2010.

[13] C.-L. Chang, H. S.-J. Lee, and C.-K. Chen, "Nucleophilic cleavage of crosslinked polysiloxanes to cyclic siloxane monomers: Mild catalysis by a designed polar solvent system," J. Polym. Res., vol. 12, no. 6, pp. 433-438, 2005.

[14] D. Oldfield and T. Symes, "Long term natural ageing of silicone elastomers," Polym. Test., vol. 15 , no. 2 , pp. 115-128, 1996. 
[15] R. F. Shepherd, A. A. Stokes, J. Freake, J. Barber, P. W. Snyder, A. D. Mazzeo, L. Cademartiri, S. A. Morin, and G. M. Whitesides, "Using explosions to power a soft robot," Angew. Chem., Int. Ed., vol. 52, no. 10, pp. 2892-2896, 2013.

[16] S. Hamdani, C. Longuet, D. Perrin, J.-M. Lopez-cuesta, and F. Ganachaud, "Flame retardancy of silicone-based materials," Polym. Degrad. Stab., vol. 94, no. 4, pp. 465-495, 2009.

[17] Shin Etsu, "Characteristic properties of silicone rubber compounds," datasheet, Mar. 2005 [Revised Jun. 2012].

[18] C. Majidi, R. Kramer, and R. Wood, “A non-differential elastomer curvature sensor for softer-than-skin electronics," Smart Mater. Struct., vol. 20, no. 10, p. 105017, 2011.

[19] Y.-L. Park, B.-R. Chen, and R. J. Wood, "Design and fabrication of soft artificial skin using embedded microchannels and liquid conductors," IEEE Sens. J., vol. 12, no. 8, pp. 2711$2718,2012$.

[20] D. Vogt, Y.-L. Park, and R. Wood, "Design and characterization of a soft multi-axis force sensor using embedded microfluidic channels," IEEE Sens. J., vol. 13, no. 10, pp. 40564064, 2013. 


\section{Supplemental Information}

\section{A Resilient, Untethered Soft Robot}

Michael T. Tolley ${ }^{1,2}$, Robert F. Shepherd ${ }^{3,4}$, Bobak Mosadegh ${ }^{2,3}$, Kevin C. Galloway ${ }^{1,2}$, Michael Wehner $^{1,2}$, Michael Karpelson ${ }^{1,2}$, Robert J. Wood ${ }^{1,2, *}$, and George M. Whitesides ${ }^{2,3, *}$

${ }^{1}$ School of Engineering and Applied Sciences, Harvard University

60 Oxford Street, Cambridge, MA 02138

${ }^{2}$ Wyss Institute for Biologically Inspired Engineering, Harvard University

3 Blackfan Circle, Boston, MA 02115

${ }^{3}$ Department of Chemistry and Chemical Biology, Harvard University

12 Oxford Street, Cambridge, MA 02138

${ }^{4}$ School of Mechanical and Aerospace Engineering, Cornell University

105 Upson Hall, Ithaca, NY 14853

*Corresponding authors,

email: gwhitesides@gmwgroup.harvard.edu

email: rjwood@eecs.harvard.edu 


\section{Experimental Methods}

To fabricate the body of the soft robot, we mixed batches of the rubber composite by blending $0.15 \mathrm{~kg}$ of hollow glass spheres, hgs ( $\rho_{\text {hgs }} \sim 0.13 \mathrm{~kg} / \mathrm{L}$; Microbubbles; $3 \mathrm{M}$ ) into $1.75 \mathrm{~kg}$ of M4601A silicone ( $\rho_{\mathrm{hgs}} \sim 1.2 \mathrm{~kg} / \mathrm{L}$ ) using a rotational mixer and impeller blade. After mixing for 30 minutes, we added M4601B catalyst to the mixture at a 1:9 ratio of M4601B:M4601A by weight. After mixing for another 10 minutes, we poured the silicone over the laser cut mold.

Though foams are an option to reduce the weight of the body, silicone foam prepolymers are not (to our knowledge) readily available at lab scale, it is difficult to predict the final volume of foam expansion, and available foam prepolymers (e.g., urethanes) have been difficult to bond to silicone in our lab-scale processes.

\section{Power Source Evaluation}

We considered the use of compressed gas to power our pneumatic soft robot. Assuming isothermal expansion at temperature $T$ (i.e. the process is slow enough for energy from the environment to heat the expanding gas), the maximum work $w$ that can be done by $n$ moles of gas at a working pressure, $p_{w}$, expanding to atmospheric pressure, $p_{a t m}$, is given by Equation 1, where $R$ is the Boltzmann constant.

$$
w=n R T \ln \frac{p_{w}}{p_{a t m}} \quad \text { (Equation 1) }
$$

For a working pressure of $16 \mathrm{psig}(214 \mathrm{kPa})$, at $20^{\circ} \mathrm{C}$, a mole of compressed gas has the potential to do $1.83 \mathrm{~kJ}$ of work. Compressed air at the commonly available pressure of 2,900 psig (20 MPa) and $20^{\circ} \mathrm{C}$ has a molar volume of $8.04 \mathrm{kmol} / \mathrm{m}^{3}$. Pressurized carbon dioxide, however, is commonly at $850 \mathrm{psig}(5.9 \mathrm{MPa})$, and has a molar volume of $17.8 \mathrm{kmol} / \mathrm{m}^{3}$. Thus, the energy density of commonly available liquid $\mathrm{CO}_{2}$ is approximately 2.2 times that of gaseous compressed 
air. Due to this higher volumetric energy density, we used $\mathrm{CO}_{2}(1)$ as our source for compressed gas.

Using the Hagen-Pouiselle relationship (Equation 2) between pressure difference, $\Delta P$, initial flow rate of gas into a Pneu-Net volume, $Q$, the gas delivery tube length, $L$, and radius, $r$, we calculated the theoretical flow rate for compressed gas from commercially available $\mathrm{CO}_{2}$ cylinders. For compressed CO2 regulated to16 psig (214 kPa) flowing through a $1 \mathrm{~m}$ tube with a $2.5 \mathrm{~mm}$ radius, the initial flow rate is $0.12 \mathrm{~m}^{3} / \mathrm{s}$. However, this value will drop rapidly as the actuator begins to pressurize. The available volume of gas from a cylinder capable of holding 44 grams of liquid $\mathrm{CO}_{2}$ (a size compatible with our larger robot design) is $\sim 10.5 \mathrm{~L}$ at the working pressure.

$$
\Delta P=\frac{8 \mu L Q}{\pi r^{4}} \quad(\text { Equation } 2)
$$

Mini air compressors (MACs) are relatively light weight $(<0.5 \mathrm{~kg})$ diaphragm pumps driven by electrical motors. They can be operated by electrical wire from a remote location, tethered operation, or via battery in untethered operation. While tethered (using thin/light copper wires), the robot can be actuated indefinitely. Two motors powered via a 3,200 mAh lithium-polymer (Li-Po; $\sim 0.5 \mathrm{~kg}$ ) can operate continuously for 1.6 hours (the motors draw $\sim 1,000 \mathrm{~mA}$ of current each). However, the mini air compressors have limited flow rates: a maximum of $11 \mathrm{~L} / \mathrm{min}$ $\left(1.8 \times 10^{-4} \mathrm{~m}^{3} / \mathrm{s}\right)$ unrestricted, or $2 \mathrm{~L} / \mathrm{min}\left(3.3 \times 10^{-5} \mathrm{~m}^{3} / \mathrm{s}\right)$ at $16 \mathrm{psig}(214 \mathrm{kPa})$. Thus, over 1.6 hours, the volume of gas at the working pressure that the compressors deliver is at least $192 \mathrm{~L}$. Though the initial flow rate of gas into a Pneu-Net provided by the MACs is lower than for compressed gas cylinders, the overall volume of gas available for actuation is much greater (192 L vs. 10.5 L). In any case, flow rates quickly become limited by back pressure in the pressurized Pneu-Net. Combined with the potential for both tethered and untethered operation, MACs were 
the most attractive option for our untethered soft robot. The air compressors we ultimately chose (BTC IIS, Parker Systems) were a good compromise between cost (\$297), weight (0.34 kg), size (7.5 cm length), and gas flow rates $(2 \mathrm{~L} / \mathrm{min})$ at the chosen working pressure. It should be noted that a potential advantage of compressed gas is the ability to accelerate actuation with higher working pressures. However, this approach would require the development of materials and/or control systems capable of preventing material failures caused by steady-state exposure to such elevated pressures.

Table S1. Materials costs for the soft robot prototype.

\begin{tabular}{|l|c|c|c|}
\hline Component & Count & Unit Cost & Extended Cost \\
\hline $\begin{array}{l}\text { Body materials } \\
\quad \text { Elastosil M4601 silicone } \\
\text { rubber }\end{array}$ & $3.5 \mathrm{~kg}$ & $\$ 21 / \mathrm{kg}$ & $\$ 74$ \\
$\quad$ Hollow glass spheres & $0.3 \mathrm{~kg}$ & $\begin{array}{c}\$ 150 / \mathrm{kg} \\
(\$ 75 / \mathrm{gal})\end{array}$ & $\$ 45$ \\
& & $\$ 16$ & $\$ 16$ \\
$\quad$ polyaramid fabric & $12^{\prime \prime} \times 40^{\prime \prime}$ & $\$ 19$ & $\$ 19$ \\
$\quad \begin{array}{l}\text { Elastosil E951 silicone } \\
\text { sealant }\end{array}$ & 1 tube & & \\
$\begin{array}{l}\text { Power and control } \\
\text { components }\end{array}$ & & & \\
$\quad$ Mini air compressors & 2 & $\$ 297$ & $\$ 594$ \\
$\quad$ Valves & 6 & $\$ 43$ & $\$ 258$ \\
$\quad$ Custom Controller Board & 1 & $\$ 40$ & $\$ 40$ \\
$\quad$ Tubing and fittings & - & - & $\$ 20$ \\
\hline Total & & & $\mathbf{1 0 6 6}$ \\
\hline
\end{tabular}

\section{Controls}

A custom, lightweight controller board was designed to control the miniature air compressors and solenoid valves that actuate the soft robot. A microcontroller (ATmega168, Atmel Corporation) on the controller board contained an Arduino bootloader for uploading, storing, and executing programs to control the soft robot. 
We designed control programs to achieve two distinct modes of locomotion: an undulating gait and a walking gait (Figure S4, Video S2). The undulating gait consisted of repeated sequence of five states. 1) The rear leg PNs were actuated simultaneously for seven seconds (Figure S4 B). 2) the rear leg and body PNs were then actuated together for half a second. 3) The rear and forward legs, as well as the body PNs were all actuated simultaneously for five seconds (Figure S4 C). 4) The front legs were actuated alone for two seconds (Figure S4 D; the differential timing in actuation between the front and back legs of approximately five seconds biased the locomotion in the forward direction).5) Finally, all of the PNs vented to atmosphere for two seconds; the MACs were also turned off for this period to facilitate venting and conserve battery power (Figure S4 E).

A second program, consisting of four states caused the robot to execute a walking gait. 1) Beginning with all legs in contact with the ground (to prevent the robot from slipping backward), we caused the rear leg to actuate for four seconds, resulting in a "stance" position (Figure S4 H). 2) The actuated rear leg, as well as the front leg on the opposite side of the body, were then actuated simultaneously for four seconds to transfer both the pressurized air and the robot's weight from the rear leg to the front one (reusing pressurized air during this transfer step increased the efficiency of the robot.) 3) The robot then thrust itself forward by both depressurizing the rear leg (allowing the stored elastic energy to straighten the rear leg), while continuing to pressurize the front leg to pull the robot forward over the course of four seconds (Figure S4 I). 4) Finally, all legs depressurized for half a second to prepare the robot for the next actuation cycle. Repeating the above four steps on alternating sides of the body resulted in our walking gait. 
Figure S1. Systems diagram of the configuration of mini air compressors and valves used to drive the robot in undulation or walking gaits.

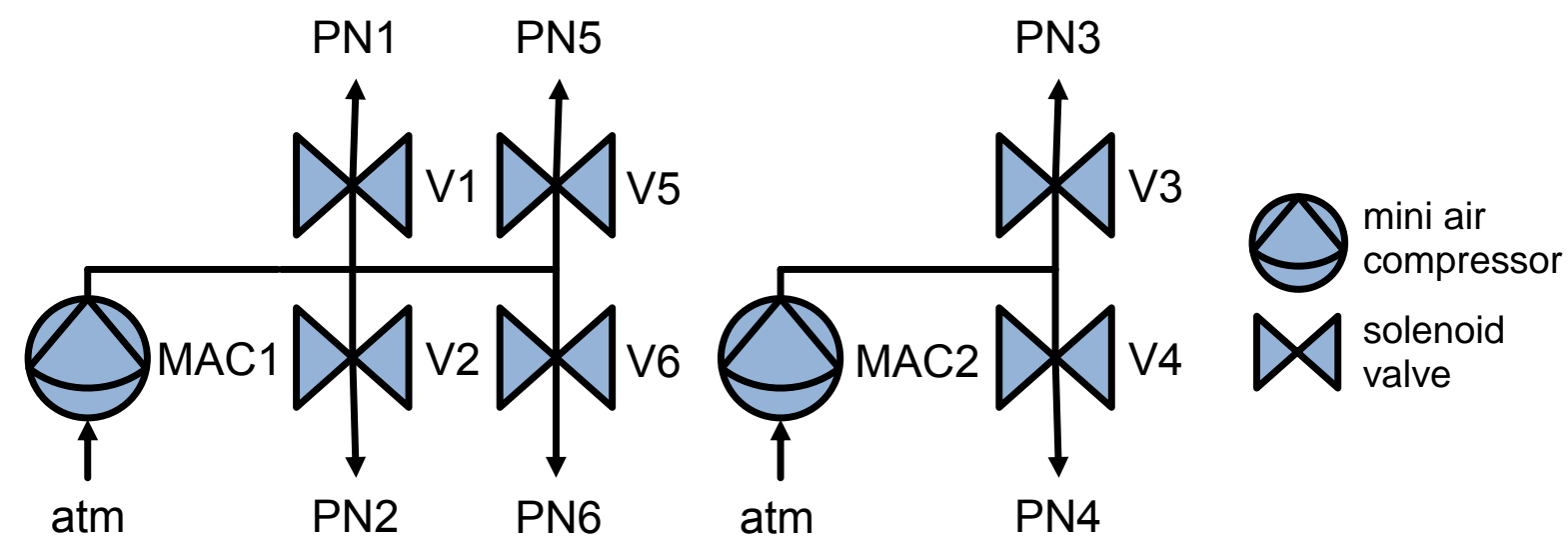


Figure S2. A The untethered robot design with the smaller tethered quadrupedal robot ${ }^{[7]}$ placed on the interior for scale. The large robot is five times longer than the small one. B Mold used to replicate the large quadruped, composed of laser-cut acrylic pieces. $\mathbf{C}$ Layer 1 of robot cast in mold with waterjet-cut aluminum pieces inserted from the top (one aluminum piece removed from the lower-right leg is shown). D Replicated Layer 1 with molded features to increase surface area and improve bonding with Layer 2. E Cut polyaramid fabric being impregnated with elastomer to form Layer 2. F A patch of elastomer-impregnated polyaramid fabric added to the ends of the limbs prevents undesired expansion at these locations.
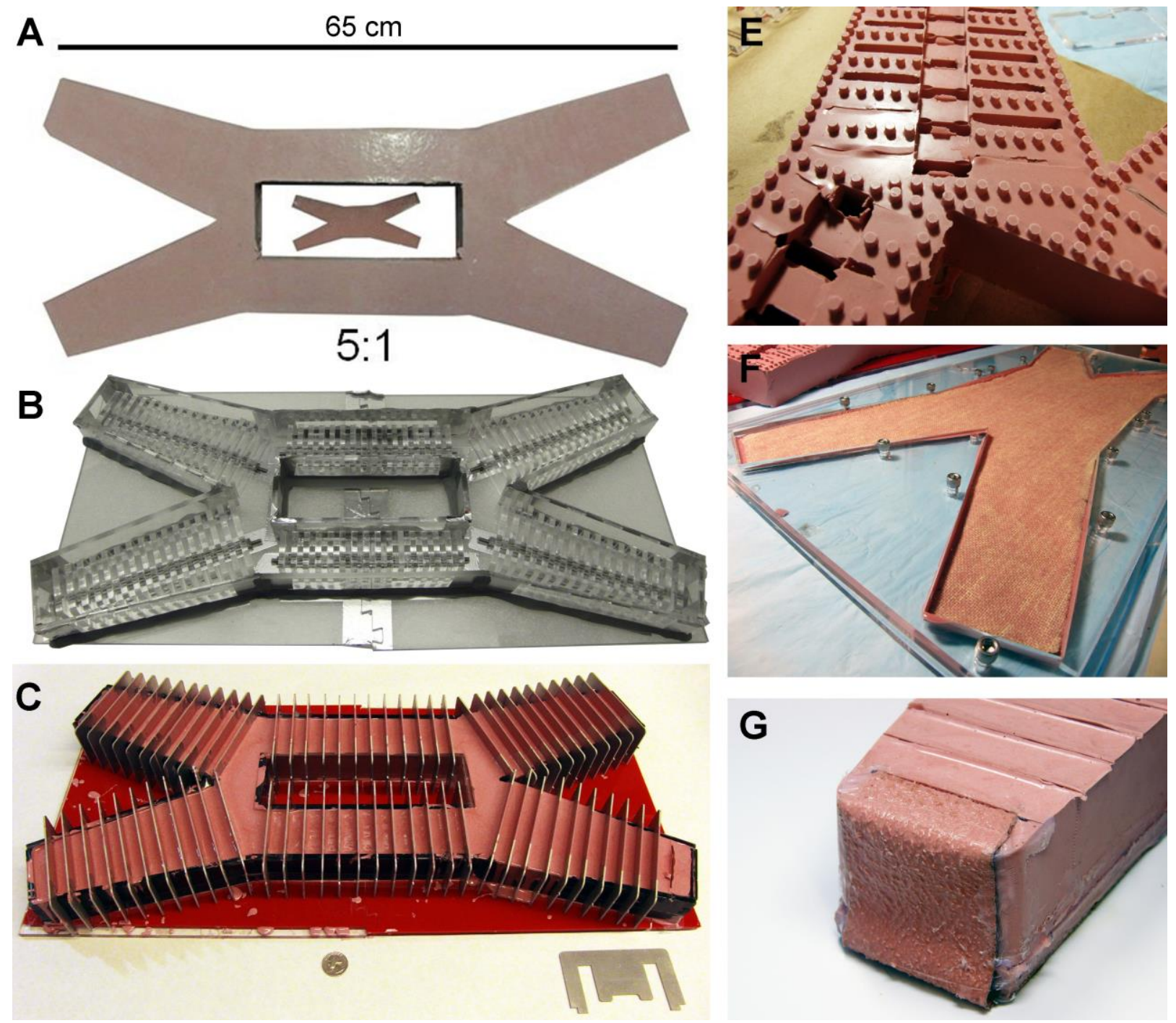

Figure S3. Maximum lift and hold tests. A Starting from a flat position, a tethered version of the soft robot was able to lift a mass of $3.4 \mathrm{~kg}(7.5 \mathrm{lbs})$ when actuated with a pneumatic pressure of $139 \mathrm{kPa}(20 \mathrm{psi})$. B Starting from an actuated position with an internal pneumatic pressure of 139 $\mathrm{kPa}(20 \mathrm{psi})$, the robot was able to hold $8.0 \mathrm{~kg}(17.6 \mathrm{lbs})$.
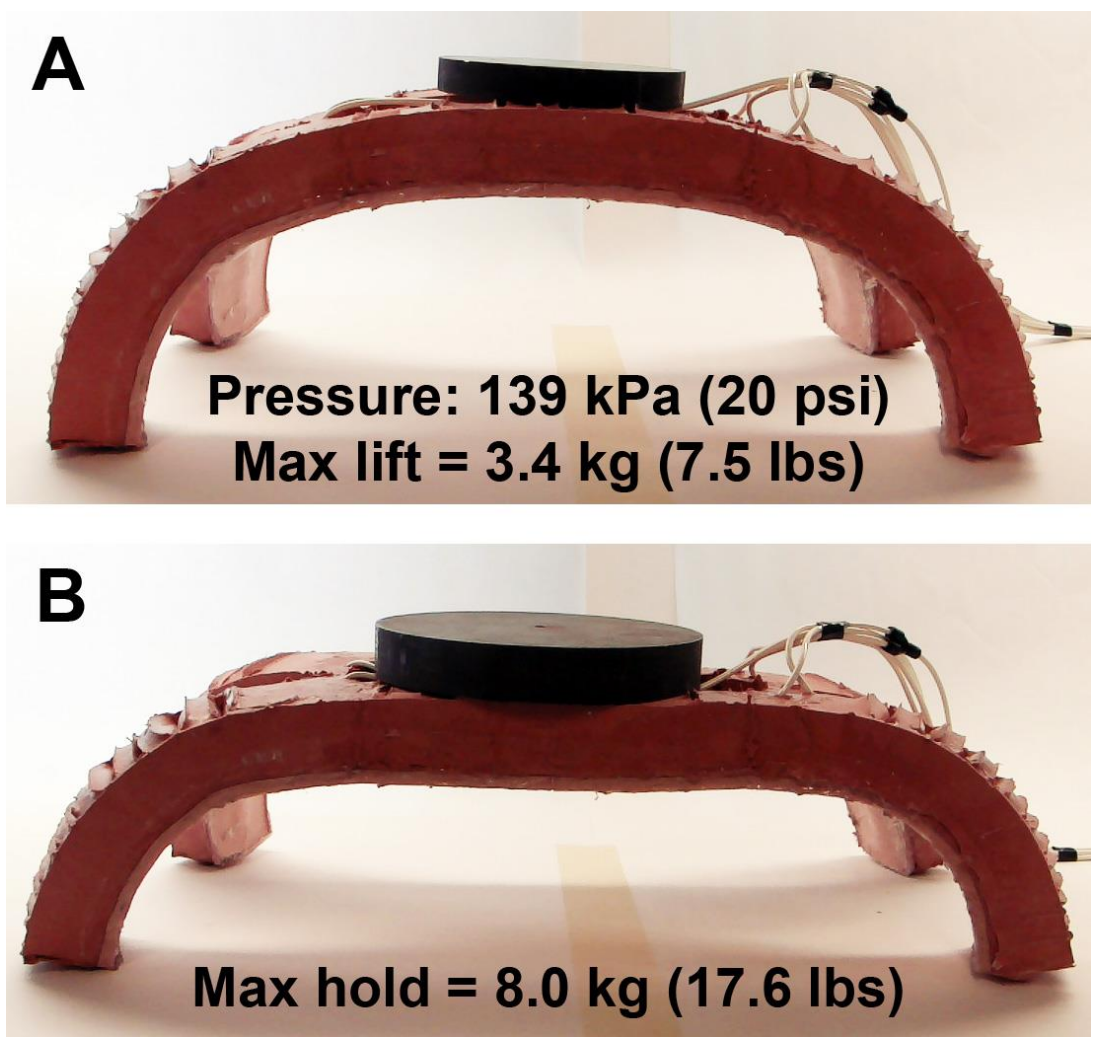
Figure S4. Frames from movies of the untethered soft robot executing undulating an ambulating gaits. A-F Undulating gait: The pneumatic channels are inflated sequentially from the rear of the robot toward the front, resulting in forward motion. Dotted lines mark the starting position for reference. G-J Ambulating gait: Starting from rest $(\mathbf{G})$, a rear leg is actuated $(\mathbf{H})$, the opposite front leg is then actuated to shift the weight forward (I). This sequence is repeated on alternating sides, resulting in a straight ambulatory gait $(\mathbf{J})$. Green dots on the figures in the upper-right corner of each frame indicate which PNs are currently actuated (pressurized), red dots indicate unactuated PNs. The time elapsed since the start of the gait is indicated on each frame.

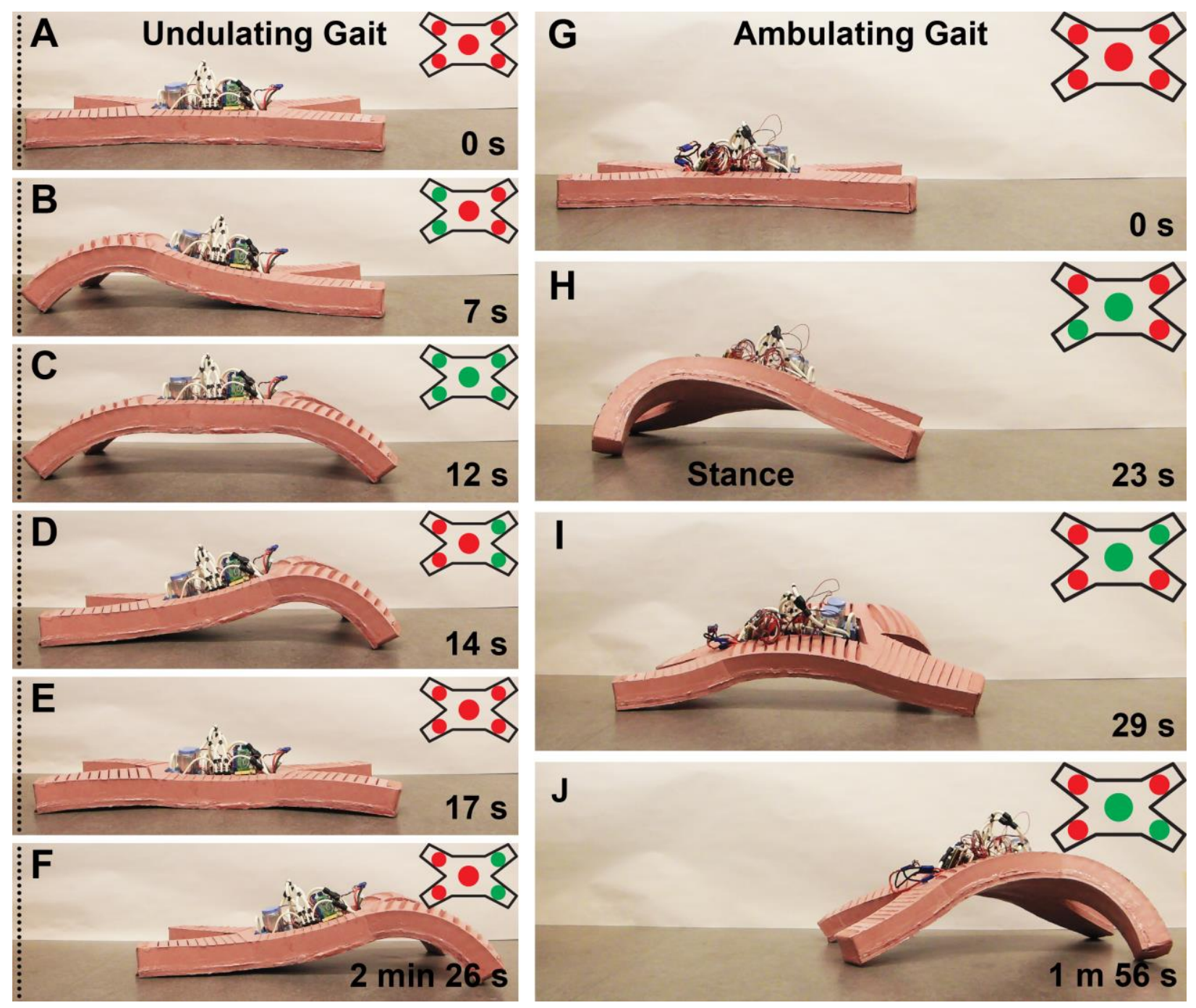


Video S1. Operation in extreme conditions. In this video we demonstrate the operation of our robot in four cases of extreme conditions: In snow, in fire, in water, and subjected to extreme crushing force. In all cases except in fire, the robot is operated untethered.

Video S2. Locomotion gaits. This video shows the soft robot locomoting untethered using two unique gaits: an undulating gait and an ambulating gait.

Video S3. Exploration mission. This video shows the untethered soft robot conducting a reconnaissance mission in a laboratory environment. Following a preprogrammed control sequence, the robot walks forward then turns left to walk into a small passageway. A forward facing camera (view overlaid in the lower-left corner of the screen) allows remote video recording. 